\title{
Fault isolation in discrete event systems by observational abstraction
}

\author{
Dan Lawesson, Ulf Nilsson, Inger Klein \\ Division of Automatic Control \\ Department of Electrical Engineering \\ Linköpings universitet, SE-581 83 Linköping, Sweden \\ WWW: http://Www. control.isy.liu.se \\ E-mail: danla@ida.liu.se, ulfni@ida.liu.se, \\ inger@isy.liu.se
}

5th October 2004

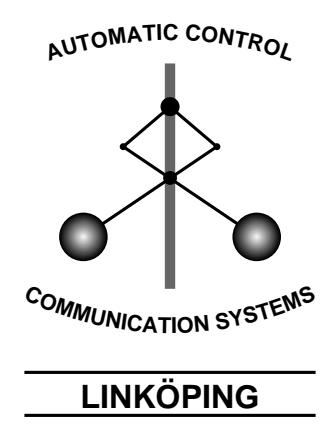

Report no.: LiTH-ISY-R-2638

Submitted to CDC 2003

Technical reports from the Control \& Communication group in Linköping are available at http://www.control.isy.liu.se/publications. 


\begin{abstract}
We propose a method for fault isolation in discrete event systems such as object oriented control systems, where the observations are the logged error messages. The method is based on automatic abstraction that preserves only the behavior relevant to fault isolation. In this way we avoid the state space explosion, and a model checker can be used to reason about the temporal properties of the system. The result is a fault isolation table that maps possible error logs to isolated faults, and fault isolation thus reduces to table lookup. The fault isolation table can also be used as an analysis tool at the design level to find both faults that cannot be isolated as well as redundant error messages.
\end{abstract}

Keywords: Fault isolation, automatic abstraction, Discrete Event Dynamical Systems 


\section{Fault isolation in discrete event systems by observational abstraction}

\author{
Dan Lawesson, Ulf Nilsson \\ Dept of Computer \& Information Science \\ Linköping University \\ 581 83, Linköping, Sweden \\ \{danla, ulfni\}@ida.liu.se
}

\author{
Inger Klein \\ Department of Electrical Engineering \\ Linköping University \\ 58183 Linköping, Sweden \\ inger@isy.liu.se
}

\begin{abstract}
We propose a method for fault isolation in discrete event systems such as object oriented control systems, where the observations are the logged error messages. The method is based on automatic abstraction that preserves only the behavior relevant to fault isolation. In this way we avoid the state space explosion, and a model checker can be used to reason about the temporal properties of the system. The result is a fault isolation table that maps possible error logs to isolated faults, and fault isolation thus reduces to table lookup. The fault isolation table can also be used as an analysis tool at the design level to find both faults that cannot be isolated as well as redundant error messages.
\end{abstract}

\section{INTRODUCTION}

In this paper we describe a model-based [8] approach to fault isolation in large object oriented control systems. The work is motivated by a real industrial robot control system developed by ABB Robotics. The system is large (the order of $10^{6}$ lines of code), concurrent, has an object oriented architecture and is highly configurable, supporting different types of robots and cell configurations. Since the system is time- and safety-critical the first priority, in case of a failure, is to bring the system to a safe state; alarms that go off are logged and can be analyzed when the system comes to a stand-still. We consider here primarily hardware faults, but we assume that the faults show up indirectly in the software. In addition we make the common single fault assumption, i.e. that a system failure is caused by only one fault (but resulting in cascading alarms).

The log thus contains partial information about the events that took place at the approximate time of the system failure. However, the order in which messages are logged does not necessarily reflect the way error messages propagate - the system is concurrent and safety critical actions may have to be taken before error reporting takes place. Hence, in what follows we (somewhat pessimistically) view the $\log$ as a set of error messages. In addition a system may contain a number of critical events that are unobservable, but which may explain all observable alarms.

There are two main goals with our method. First, the method can be used for operator support. The aim is then to single out the error message that explains the actual cause of the failure, or possibly an unobservable critical event explaining the observations. That is, we aim to discard error messages which are definitely effects of other error messages, while trying to isolate error messages (or critical events) which explain all other messages. Second, our method can be used at the design level. At the design level, we want to find out if the error log design is sufficient, that is, if enough error messages are produced to be able to isolate all faults.

Given the size of the software it is not possible to use the code directly - we have to rely on a model of the software. The modeling language used here is part of the Unified Modeling Language (UML) [15]. UML is a design notation for object oriented systems and also serves as a system documentation. The main reason for using a UML model is that developing such a model is an integrated part of the software development, and thus the model can be obtained and maintained at a low extra cost. In our approach we use UML class diagrams, collaboration diagrams and state chart notation.

In standard AI diagnosis literature, see e.g. [13], a diagnosis is a (minimal) set of failed components explaining the observations. For dynamic systems (systems with state), however, a diagnosis is often defined as the set of all runs, or trajectories, consistent with the observations (see e.g. [6], [5]). This definition is generally insufficient to isolate the origin of the fault(s), and requires post-processing to pinpoint e.g. the faulty component(s). Our approach is more direct and focuses on finding the alarm that explains (is consistent with) all observables: given the system description, and the observations, we try to infer the origin of the fault using properties of events, expressed in a specification language based on a subset of the temporal logic CTL, originally developed for verification [4].

Our approach also bears some resemblance to that of Sampath et al. [16]. However their work is mainly concerned with diagnosability in discrete event systems; i.e. to detect, within finite delay, whether a certain type of fault has occurred. While we focus on post-mortem analysis, the work reported in [16] is mainly intended for monitoring and online detection and diagnosis.

The problem we address is similar to the problem treated in [2]. They study networked systems with distributed sensors that report alarms to a global supervisor. There is no global time so the supervisor should act on a partially ordered set of alarms, and they propose the use of net unfoldings in Petri nets.

We use model checking [4] to perform the temporal reasoning. Typically used for system design verification, a model checker evaluates a temporal logic formula for a given system specification. Properties of the design such as liveness 
(the system is guaranteed to perform certain behaviors) and safety (some unsafe system states will never be reached) can be checked.

Model checking suffers from the state-space explosion problem, i.e. the fact that the state space grows exponentially with the size of the system description. Several approaches to this problem have been proposed, mainly symbolic model checking and partial order reduction.

Symbolic model checking [11] reasons about sets of states and represents them in an implicit form using e.g. boolean formulae. The representation of a formula that holds for a certain set of states may be much smaller than the set itself. Partial order reduction [7], [12] abstract away from details about ordering of transitions whose relative ordering has no influence on the temporal logic formula being checked. The two approaches were successfully combined in [1].

By restricting the application domain the abstraction can be made stronger than in partial order reduction. In our case we do not solve a general model checking problem but a more specific problem. Therefore, there are more efficient abstraction mechanisms for our particular problem, and we propose such a method in this paper.

The general idea is as follows. Since we are only interested in the correlation between the first critical event and the set of messages that are logged during the execution, we can abstract away details not only about parallel object interleavings as in partial order reduction, but also ignore order of messages and dynamics that in the global system model does not change the set of messages sent or the order of critical events. For example, cyclic behavior where no critical events occur can be abstracted to a single state.

The result produced by our method is a table that maps all possible message logs to the corresponding strong root candidates. A strong root candidate is an event that is known to have occurred, and there is a run (consistent with the log) where this event is the first critical event. The table is called the fault isolation table and can obviously be used for fault isolation. Given a $\log$ and the fault isolation table, the strong root candidates can be found by table lookup. The primary use is in diagnosability analysis, though. The table partitions all possible system runs in equivalence classes of runs with the same logged messages. Each partition corresponds to a row in the table. If for such a row, there are several strong root candidates, we conclude that runs in the corresponding class are not diagnosable. If an error message is redundant, it will be evident from the table. If it is dependent on some other message, the two will only appear in certain configurations in consistent logs. The exponential size of the table indicates that it is not feasible to use it explicitly in general for systems with a large set of logged events. Then, abstractions of the table can be considered and presented to a user, for example the set of table rows that indicate nondiagnosability.

We have developed a prototype tool, StateTracer, that takes a description of a system as input and produces a fault isolation table as output along with vizualisations of all merged objects. The system description is given in UML.

The rest of the paper is organized as follows. In Section II we outline the notation and theory used for the rest of the paper. Automatic abstraction of the state space of a system is described in Section III, followed by a description of our prototype tool in Section IV and an example in Section V. Finally, Section VI concludes the paper with a summary.

\section{THE MODEL OF A SYSTEM}

In this section we outline the mathematical foundation of our work, see [10] for details.

A system consists of a finite set of inter-connected objects, where each object has a finite set of states and a transition relation describing the state changes of the object. State changes are triggered by events. There are five types of events: epsilon, sending, receiving, logging and critical events. Epsilon events are internal events, i.e. events that need no synchronization with other events to be enabled, that are not observable outside of the object. Sending events synchronize with a receiving event in a connected object. Logging events are internal events that produce a message to the log when taken, and critical events are internal events that are associated to a cause of system failure.

Typically, enabled transitions are considered time-outs and will take place eventually if enabled. The exception from this rule are transitions that are labeled optional. Optional non-synchronized events can be used to model events that are triggered by some entity in the world that is not in the model. Critical events are always considered optional.

The system semantics associates a given system with a set of runs. A run is a sequence of (global) states and in each such state every object of the system is assigned a (local) state. The states of each object are instrumented with labels, indicating which logged and critical events that have taken place prior to reaching the state.

To facilitate reduction in the state space of the system we propose the following abstraction of a run. Let $t$ be a run, then $\operatorname{obs}(t)=(e, S)$ where

- $e$ is the first critical event of $t$,

- $S$ is the set of all critical and logged events of $t$.

Note that by definition the set of logged and critical events in a run is growing monotonically, and stabilizing after a finite number of steps (due to finiteness of the number of objects and object state transitions). Now the model of a system is the set of all obs $(t)$ for all runs $t$ of the system. The model is more abstract than the set of runs, yet it contains enough information to establish the relation between logged messages and critical events which is enough to perform fault isolation.

\section{A. Observational equivalence}

We say that a set of objects $O$ is observational equivalent to an object $o$ in a system (description) $S D$ if $o$ can be 
exchanged for $O$ in $S D$ such that the model of $S D$ remains unchanged. For our purpose we may replace a system model by a simpler model without altering the fault isolation table as long as observational equivalence is preserved.

1) Cartesian product: Given a system and a pair of objects in the system, we can construct an object $o$ that can replace the two without changing the model of the system. Some of the states of $o$ may not be reachable and may then be removed. The resulting reachable set of states constitutes the Cartesian product of the two original objects.

2) Forced epsilon and log removal: Assume that an object has a state $s$ from which there is only one transition $t$ and it will eventually be taken in any run reaching $s$. Then $s$ can be removed from the object, and in-going transitions moved to the target state of the transition without changing the model of the system in which the object is located. Logged events and critical events are kept in a history component of the local states, and thus transitions need not be kept to maintain observational equivalence.

3) Epsilon and log cycle merging: Assume that there is a cycle of internal transitions in an object. The states on this cycle can all be merged into a single state $s$ without changing the model of the system the object belongs to. In order to model the case where some transitions can be refused on the cycle, i.e. transitions that are enabled only in a subset of the states of the cycle, the transitions from $s$ are labeled to indicate whether they are optional or not. An optional transition from $s$ corresponds to a transition enabled only in a strict subset of the states on the cycle.

\section{Automatic ABStraction}

The concepts described above show how the state space of the system can be reduced without altering the model of it. We propose an incremental approach where the objects of the system are gradually merged into Cartesian products and after each merge the state space of the new object is reduced by forced epsilon and log removal and epsilon and log cycle merging. We merge the smallest connected objects first and continue until all objects of the system have a state space size larger than a given threshold $\max$. Then the abstracted system is analyzed by the model checker.

\section{A. Fault isolation terminology}

We use the term scenario to denote a model and a message $\log$ from a system that has failed. Given such a scenario we would like to pinpoint the fault that has caused the failure of the system. We are interested in the first critical event to occur in the scenario and call this event the root event.

We use the term present for system events that are known to have occurred. A system event $e$ is called enabled root if there is a run of the system in which $e$ is the first critical event and the run is consistent with the message log.

A strong root candidate is an event that is both present and enabled. Having exactly one strong root candidate we assume that we have found the root event. Else, the scenario is considered not diagnosable.

See [9] for a more elaborate description of these concepts.

\section{THE PROTOTYPE TOOL}

We have developed a prototype tool, StateTracer, that takes a description of a system as input and produces a fault isolation table as output along with vizualisations of all merged objects. The system description can be formulated in a textual language, but StateTracer also integrates as a plug-in with the UML modeling tool Argo/UML [14], adding an extra menu for computing a fault isolation table of the currently modeled system.

There are columns in the table for each possible log message and two additional columns for corresponding enabled roots and strong root candidates. See Table I for an example.

After appropriate model abstraction, the table is computed using the model checker NuSMV [3] in a four phase algorithm.

1) First, all rows in the table are tested for consistency with the observations given for the row in question. Table rows that correspond to inconsistent logs are skipped in the future and not even reported to the user. The consistency check is performed by one invocation of NuSMV with $2^{n}$ specifications, one for each row.

2) Next, all consistent rows are tested for criticality consistency, i.e. ensuring that the $\log$ is consistent with the assumption that at least one critical event has taken place.

3) In the third invocation of NuSMV, the set of enabled roots is calculated. This is done by one specification for each critical event $e$ and critical consistent $\log L$, testing if there is a run where $e$ is the first critical event to take place, and eventually the run produces a log that is equal to $L$.

4) In the last phase the set of present critical actions is computed. For each critical consistent $\log L$ and critical event $e$, one specification is created that computes if $e$ takes place in all runs that correspond to $L$.

\section{EXAMPLE}

As an example, see Figure 1, we consider a motion control system for an industrial robot. The control system uses a bus for communication with the servo, and the bus is also used by a server application that is not in any other way connected to the control system. It is included because its clients discover faults of the bus as a side effect of their normal operation which we for brevity do not model in the example.

The system is modeled in five singleton classes, Moc, Ipol, Servo, Bus and Server that are instantiated in exactly one object each. The class Client has four instances. Thus, there are 9 objects in the system. See Figure 2 for the transitions relations for three of the classes as output from StateTracer. StateTracer uses an exclamation mark for sending events 


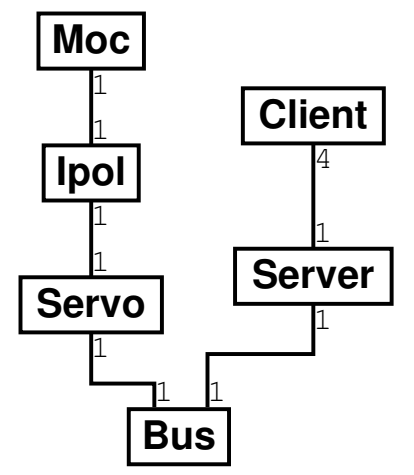

Fig. 1. The static structure of the example system.

and question mark for receiving. Logged events are prefixed with log: and crit: is used as critical event prefix. Optional transitions are indicated by empty arrow heads.

In normal operation, Clients make requests to the Server that uses the Bus to serve the requests. Moc sends position instructions to Ipol which computes segments that are used to control the Servo which in turn relies on the Bus to function correctly.

After merging moc and ipol, servo is added, resulting in a Cartesian product $o$ of all three objects. Assume no abstraction was made and the Cartesian product was computed naively. Then $o$ would have $3 * 4 * 7=84$ states and three independent boolean history variables indicating which critical and logged events have taken place (the labeling of the states), resulting in a state space size of 672 . Using our proposed abstraction scheme we get the state space size of 6 states as shown in Figure 3. The boolean history variables are attributed to the states and names of boolean variables that hold true are shown in respective states.

The resulting fault isolation table is given in Table I. Logged error messages from the four Client instances are called c1,c2,c3 and c4 whereas instances of singleton classes are named after the class name. Since there are 5 logged messages in the system, the table could have as many as 32 rows. The messages from Client instances are dependent on each other since if one object logs, the other ones are also forced to log. StateTracer only reports the rows corresponding to consistent runs, and thus, the reported table has only 4 rows.

To get optimal performance in terms of total execution time, there is a trade-off between spending a short time merging objects and providing a small state space for the model checker to deal with. Table II gives a hint of the complexity of reaching optimality. $T_{a}$ is the abstraction run time and $T_{m}$ the model checking time. $N$ is the number of objects remaining after abstraction, and Reach is the number of reachable states. We used different thresholds $\max$ for when to stop automatic abstraction and start model checking. In the first case we did no abstraction at all, and in the
TABLE II

RUN TIMES FOR THE EXAMPLE.

\begin{tabular}{|r|r|r|r|r|r|}
\hline $\max$ & $T_{a}$ & $T_{m}$ & Reach & StateSpace & $N$ \\
\hline \hline 0 & 0.00 & 6.13 & 5765 & 2211840 & 9 \\
10 & 0.01 & 1.54 & 1934 & 39936 & 5 \\
40 & 0.02 & 2.16 & 1719 & 29952 & 4 \\
50 & 0.08 & 5.71 & 1504 & 22464 & 3 \\
140 & 13.48 & 0.88 & 137 & 137 & 1 \\
\hline
\end{tabular}

last case we continued merging until the entire system is represented by a single automaton.

Not surprisingly, the time for abstraction increases quickly with the number of objects merged - we perform more merge steps and objects being merged are larger. Clearly, the size of both the state space and the set of reachable states shrinks monotonically with increasing threshold, but the relationship between model checking running time and state space size is more complex. There are two major reasons for this.

1) The model checker is a symbolic model checker that does not operate on an explicit representation of the state space, but rather on boolean formulae that represent a set of states. The size of these formulas does not correlate well with the size of the set they represent.

2) When abstraction is stopped before abstracting the entire state space into a single object, the objects tend to have large state transition relations. Thus, the state space size is reduced by abstraction, but the number of transitions of the system may not have decreased to the same extent. This increases the time for generation of the specification as well as parsing the specification and building the transition relation BDD.

Furthermore, merging small objects is cheap whereas the model checker considers the global Cartesian product of the state space and is therefore helped by abstraction which is shown by comparing the total run time $\left(T_{a}+T_{m}\right)$ of the two first rows in Table II.

We have found that systems with strong dependence between objects, as the motion control part of the example system where for example the interpolator controls the servo and thus imposes a strong correlation between states of the two objects, is well suited for abstraction. In such a situation the system can be represented by a highly abstract version that can be model checked more efficiently.

On the other hand, if there is a high degree of independence between objects that may trigger critical events, such as a peer-to-peer network, the model checker will not benefit much from abstraction. It may be noted that if the parallelism does not affect the diagnosis, e.g. interleaving of logged messages, the model is efficiently abstracted as soon as the parallelism is completely contained in one single object.

\section{CONCLUSIONS}

We have shown that the state space of the system can be explored without suffering the penalties of state space explosion by abstracting the details of object inter-communication 

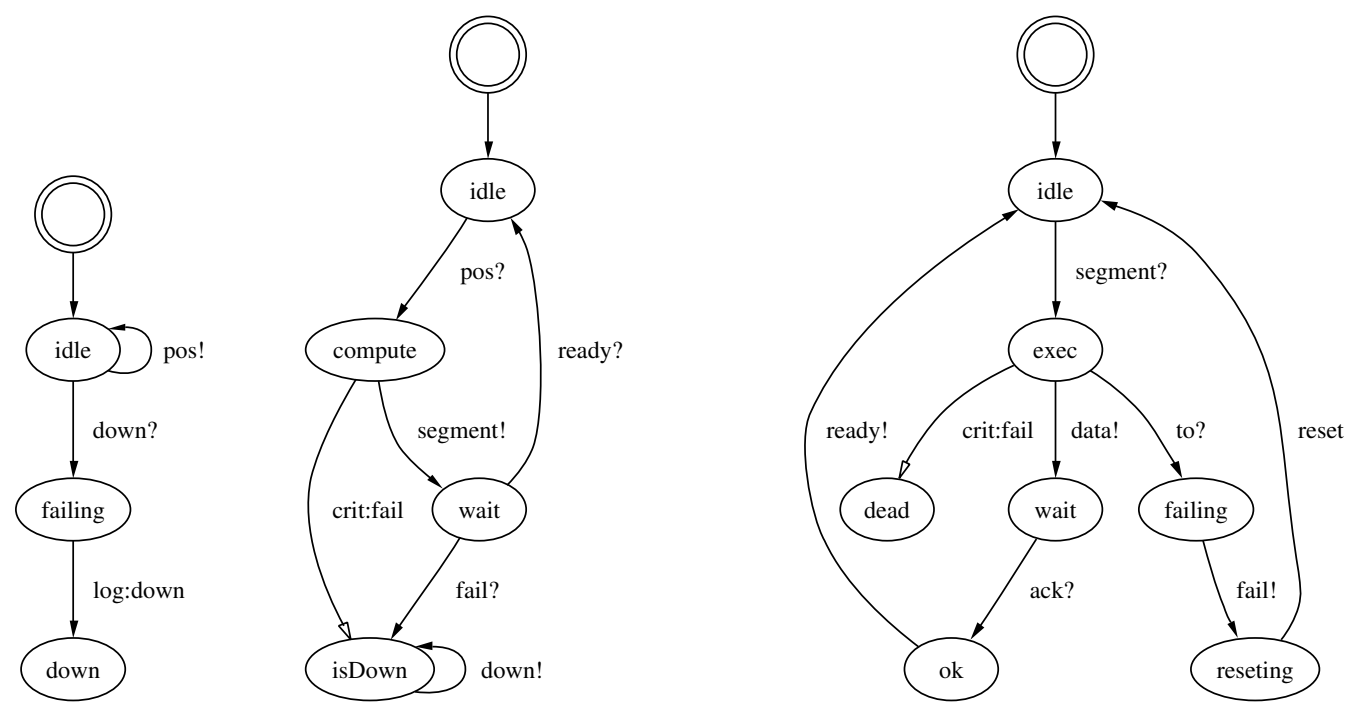

Fig. 2. Transition relations for (from left) classes Moc, Ipol and Servo.

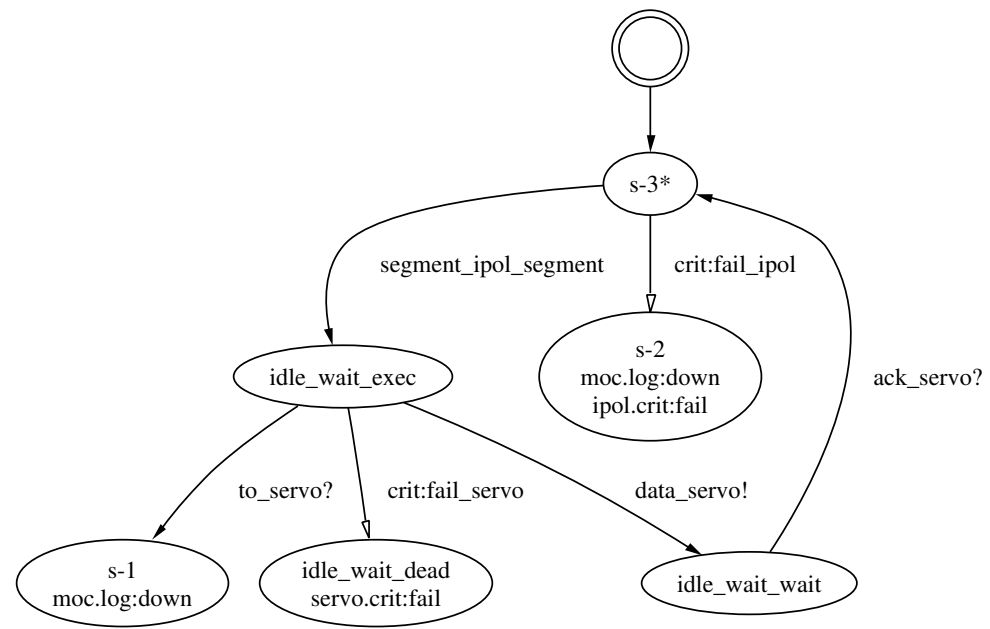

Fig. 3. Transition relation for the abstracted version of Moc, Ipol and Servo. The first line of text in a state is the name, followed by one line for each boolean history variable that holds true in the state. Some long state names are abbreviated to s- $n$ for layout reasons. A star following the state name indicates a transient state, i.e. a state with an outgoing non-optional epsilon transition. 
TABLE I

THE RESULTING FAULT ISOLATION TABLE FOR THE EXAMPLE SYSTEM.

Logged events

Fault isolation results

\begin{tabular}{|c|c|c|c|c||l|l|}
\hline c1 & c2 & c3 & c4 & moc & Enabled Roots & Strong Root Candidates \\
\hline & & & & & servo.fail & servo.fail \\
\hline X & X & X & X & & bus.down, servo.fail & bus.down, servo.fail \\
\hline & & & & X & ipol.fail & ipol.fail \\
\hline X & X & X & X & X & bus.down, ipol.fail & bus.down \\
\hline
\end{tabular}

and interleaving of events that are not of interest in our case of fault isolation.

Details about system behavior in the original system model that are not relevant to the fault isolation table are abstracted away in an incremental process. Internal state transitions within objects that can be proven not to contribute to the fault isolation table are removed by forced epsilon removal and epsilon cycle merging. Irrelevant inter-object communication dynamics is removed by making the communication internal in a new object that simulates a set of objects.

The proposed approach allows for automatic abstraction to be used for small objects where it performs well and when a certain limit of object size is reached, the system is analyzed by a model checker.

\section{ACKNOWLEDGMENTS}

This work has been financially supported by VINNOVA's Center of Excellence ISIS - Information Systems for Industrial Control and Supervision. We are also grateful for the cooperation with $\mathrm{ABB}$ Robotics, and in particular Magnus Larsson.

\section{REFERENCES}

[1] R. Alur, R. Brayton, T. Henzinger, S. Qadeer, and S. Rajmani. PartialOrder Reduction in Symbolic State Space Exploration. In $C A V$, pages 340-351, 1997.

[2] A. Benveniste, E. Fabre, C. Jard, and S. Haar. Diagnosis of Asynchronous Discrete Event Systems, a Net Unfolding Approach. IEEE Trans. on Automatic Control, 2003. To appear.

[3] A. Cimatti, E. M. Clarke, E. Giunchiglia, F. Giunchiglia, M. Pistore, M. Roveri, R. Sebastiani, and A. Tacchella. NuSMV 2: An OpenSource Tool for Symbolic Model Checking. In CAV, July 2002.
[4] E. M. Clarke, O. Grumberg, and D. Peled. Model Checking. MIT Press, 1999.

[5] L. Console, C. Picardi, and M. Ribaudo. Diagnosis and Diagnosability Analysis using PEPA. In Proc. 14th European Conference on Artificial Intelligence, pages 131-136. IOS Press, 2000.

[6] M.-O. Cordier, L. Rozé, and Y. Pencolé. Incremental Decentralized Diagnosis Approach for the Supervision of a Telecommunication Network. In Proc. 12th Intl Workshop on Principles of Diagnosis, DX01, 2001.

[7] P. Godefroid and P. Wolper. A Partial Approach to Model Checking. In Logic in Computer Science, pages 406-415, 1991.

[8] W. Hamscher, L. Console, and J. de Kleer. Readings in Model-Based Diagnosis. Morgan Kaufmann Publishers, 1992.

[9] D. Lawesson. Towards Behavioral Model Fault Isolation for Object Oriented Control Systems. Licentiate thesis no 863, Department of Computer and Information Science, Linköping University, 2000.

[10] D. Lawesson, U Nilsson, and I Klein. Model-checking Based Fault Isolation using Automatic Abstraction. Technical Report LiTH-ISYR-2493, Linköping University, 2003.

[11] K. L. McMillan. Symbolic Model Checking. Kluwer Academic, 1993.

[12] W .Penczek, R. Gerth, R. Kuiper, and M. Szreter. Partial Order Reductions Preserving Simulations. In Burkhard, H.-D., Czaja, L., Nguyen, H.-S., and Starke, P., editors, Proceedings of the CSP'99 Workshop, Warsaw, 28-30 September 1999, pages 153-171, 1999.

[13] R. Reiter. A Theory of Diagnosis from First Principles. Artificial Intelligence, 32(1):57-95, April 1987.

[14] J. Robbins and D. Redmiles. Cognitive Support, UML Adherence, and XMI Interchange in Argo/UML, 1999.

[15] J. Rumbaugh, I. Jacobson, and G. Booch. The Unified Modeling Language Reference Guide. Addison Wesley, 1999.

[16] M. Sampath, R. Sengupta, S. Lafortune, K. Sinnamohideen, and D. Teneketzis. Diagnosability of Discrete-Event Systems. IEEE trans. Automatic Control, 40(9):1555-1575, 1995. 